\title{
The SPIDER fission fragment spectrometer for fission product yield measurements
}

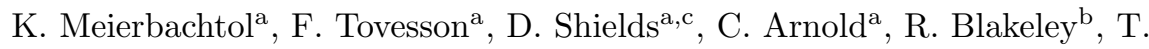 \\ Bredeweg $^{\mathrm{a}}$, M. Devlin ${ }^{\mathrm{a}}$, A.A. Hecht ${ }^{\mathrm{b}}$, L.E. Heffern ${ }^{\mathrm{b}}$, J. Jorgenson ${ }^{\mathrm{a}}$, A. \\ Laptev $^{\mathrm{a}}$, D. Mader ${ }^{\mathrm{b}}$, J.M. O’Donnell ${ }^{\mathrm{a}}$, A. Sierk ${ }^{\mathrm{a}}$, M. White ${ }^{\mathrm{a}}$ \\ ${ }^{a}$ Los Alamos National Laboratory, Los Alamos, NM 87545 \\ ${ }^{b}$ University of New Mexico, Albuquerque, NM 87131 \\ ${ }^{c}$ Colorado School of Mines, Golden, CO 80401
}

\begin{abstract}
The SPectrometer for Ion DEtermination in fission Research (SPIDER) has been developed for measuring mass yield distributions of fission products from spontaneous and neutron-induced fission. The $2 \mathrm{E}-2 \mathrm{v}$ method of measuring the kinetic energy (E) and velocity (v) of both outgoing fission products has been utilized, with the goal of measuring the mass of the fission products with an average resolution of 1 atomic mass unit (amu). The SPIDER instrument, consisting of detector components for time-of-flight, trajectory, and energy measurements has been assembled and tested using ${ }^{229} \mathrm{Th}$ and ${ }^{252} \mathrm{Cf}$ radioactive decay sources. For commissioning, the fully assembled system measured fission products from spontaneous fission of ${ }^{252} \mathrm{Cf}$. Individual measurement resolutions were met for time-of-flight (250 ps FWHM), spacial resolution (2 mm FHWM), and energy (92 keV FWHM for 8.376 MeV). Mass yield results measured from ${ }^{252} \mathrm{Cf}$ spontaneous fission products are reported from an E-v measurment.

Keywords: Spectrometer, fission mass yields, $2 \mathrm{E}-2 \mathrm{v}$ method, ${ }^{252} \mathrm{Cf}$.

\section{1. Introduction and Background}

2 Measurements of fission mass yields have been ongoing since the discovery 3 of fission [1-4]. Methods of measurement have included radiochemical separa4 tions, mass separators, and measurements with gridded ionization chambers,
\end{abstract}

Preprint submitted to Nuclear Instruments and Methods A

February 11, 2015 
commonly referred to as the $2 \mathrm{E}$ method [5]. Radiochemical separations directly measure the cumulative yields of products with high accuracy but are limited to products with half lives longer than the time scale of the separation technique. Mass separators have been used successfully to measure mass yields with high resolution but are severely limited in efficiency and are typically limited to neutron energies available at reactors $[6,7]$. The $2 \mathrm{E}$ method measures mass yields with a small, simple detector with high detection efficiency but limited in mass resolution to 3-5 amu. Presently, no technique is able to combine high resolution with high efficiency. Measurement limitations (e.g. half-life limits or only energy measurement) require assumptions to be made during analysis that leave ambiguities in final reported values and uncertainties.

The $2 \mathrm{E}-2 \mathrm{v}$ method for fission mass measurements was introduced by the COSI-FAN-TUTTI spectrometer in the 1980's at Institut Laue-Langevin in Grenoble, France [8-10]. This technique determines the mass of fission products by measuring the energy and time-of-flight over a measured path length of both fission products. In this paper fission products are defined as the products created by fission after prompt neutron and gamma emission but before betadelayed neutron emission. All results shown are post prompt-neutron emission yields. By combining measurements of energy $(E)$, time-of-flight $(t)$, and particle trajectory/flight path length $(l)$, the mass of the fission product can be determined from the classical formula for kinetic energy, solved for mass:

$$
M=\frac{2 E t^{2}}{l^{2}}
$$

High mass resolution results are achievable with this method by maximizing the resolution associated with each individual measurement. The formula for the mass resolution is:

$$
\frac{\delta M}{M}=\sqrt{\left(\frac{\delta E}{E}\right)^{2}+2\left(\frac{\delta t}{t}\right)^{2}+2\left(\frac{\delta l}{l}\right)^{2}}
$$

${ }_{16}$. Measuring both fission products in coincidence effectively measures the neu${ }_{17}$ tron emission spectrum during analysis. This is important for converting post- 

$40 \quad[17]$.

neutron emission measurements to pre-neutron emission mass distributions. The best mass resolution achieved by the COSI-FAN-TUTTI spectrometer using this method was 0.64 amu for the light fission products from ${ }^{229} \mathrm{Th}$. Other reactions measured by this spectrometer included ${ }^{239} \mathrm{Pu},{ }^{241} \mathrm{Pu}\left(\mathrm{n}_{t h}, \mathrm{f}\right)[9,11-13]$. However, the efficiency of this detector was extremely low, making measurements of fission products in the tails of the mass yield distributions a lengthy endeavour and any measurement of fissionable species with low cross sections, particularly in a survey of neutron energy dependence on yields, very difficult and time consuming. SPIDER will combine the resolving capabilities of the COSI-FAN-TUTTI spectrometer with comparably higher detection efficiencies by increasing the physical acceptance of the instrument. The need for high resolution mass yields of neutron-induced fission of actinides, in particular over a large range of incident neutron energies, has been articulated by both the theoretical modeling community and the applications community [14-16]. This paper covers the details of the detector components of the SPIDER instrument, detector testing with alpha and ${ }^{252} \mathrm{Cf}$ spontaneous fission sources to establish individual measurement resolution capabilities. Neutron-induced fission measurements are planned at the Los Alamos Neutron Science Center (LANSCE) at Los Alamos National Laboratory. Building SPIDER at LANSCE takes advantage of the expansive neutron energy range available through spallation reactions with the proton beam on two separate metal targets.. These two targets produce intense neutron beams with energies between $10^{-3} \mathrm{eV}$ and several hundred $\mathrm{MeV}$.

\section{SPIDER Instrument}

The SPIDER instrument consists of opposing detector arms (currently two) each containing two timing detectors with position-sensitive readout and an energy detector, all oriented along one central trajectory. For 2E-2v measurements the arms are paired to measure binary fragment emission, one on either side of the target. The following subsections describe each detector component 


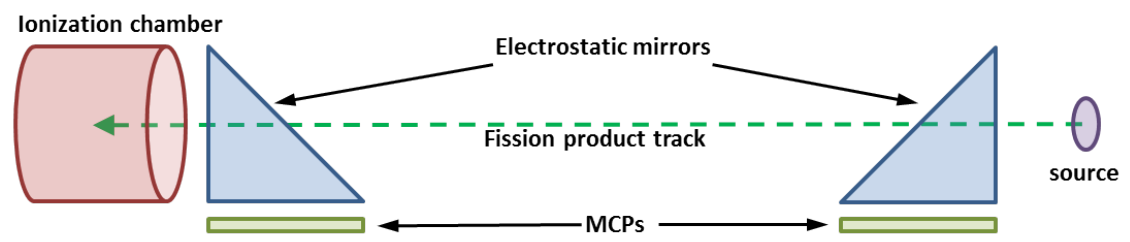

Figure 1: Cartoon of E-v detection method. Each arm in SPIDER will incorporate this method. Fission products from source shown on right travel through two timing detectors (MCPs) and an energy detector (ionization chamber).

in detail.

A diagram of one arm is shown in Fig. 1. The source or target material is placed in front of the electrostatic mirror in the first timing detector setup.

\subsection{Timing and position detection}

The two timing detectors of each arm register when a fragment passes through. The timing detectors consists of a carbon foil for production of conversion electrons via interaction with the fission product, an electrostatic mirror for directing the conversion electrons $90^{\circ}$ relative to the fission trajectory, a pair of microchannel plates (MCPs), and a delay line anode (DLA) for readout of the electronic signal from the MCPs. The MCPs are $75 \mathrm{~mm}$ in diameter and stacked a Chevron configuration. The plates amplify the signal by electron multiplication of the initial secondary electrons. The DLA has two wire pairs that provide lateral x,y position information for each event, four signals per DLA. These signals provide path length corrections for fragments traveling at angles relative to the central trajectory. This detector setup was chosen based on the fast timing characteristics of MCPs, the scalability of the MCPs to the desired diameter for the efficiency goals, and the ability to integrate position-sensitive readout which enables a detailed measurement of the particle trajectory. More detailed information about the timing and position detector components can be found in Ref. [18]. 


\subsection{Energy detection}

The energy detector for each SPIDER arm is an axial ionization chamber. Axial ionization chambers are built with the electron drift direction parallel to the trajectory of the ion. Figure 2(a) shows a schematic of the ionization chamber for SPIDER while figure 2(b) is a picture of the internal components of the ionization chamber.

The entrance window is constructed by the Norcada company [19] out of a $200 \mathrm{~nm}$ thick silicon nitride membrane. The membrane is laid down on a 200 $\mu \mathrm{m}$ thick silicon wafer. After application of the thin membrane to the wafer, a $13 \times 13$ array of $3.7 \mathrm{~mm}$ by $3.7 \mathrm{~mm}$ openings is etched into the wafer leaving only the membrane to serve as the window. Figure $3(\mathrm{a})$ is a picture of one of these windows. The transmission of fission fragments through the window array is approximately $50 \%$ relative to the MCP solid angle acceptance. The delicate structure of the silicon wafer requires an additional support structure to withstand the pressure differential of 85 Torr between the vacuum chamber and the ionization chamber. A stainless steel plate with a matching 13 x 13 array was designed to hold the silicon wafer. A layer of Torr seal epoxy was then applied to create the vacuum seal on the window. Figure 3(b) is a schematic of the window-mounting components. The entrance window structure also serves as the cathode plate of the ionization chamber. The electric field maintains a uniform drift velocity for the ionization electrons by employing ten copper guard rings $7.64 \mathrm{~cm}$ in diameter spaced $0.655 \mathrm{~cm}$ apart and connected together through $1 \mathrm{M} \Omega$ resistors, bringing the field up to negative high voltage between -2 and $-4 \mathrm{kV}$ on the Frisch grid. The Frisch grid is made of a copper mesh with 20 wires per inch $(7.9$ per $\mathrm{cm})$, permitting $95 \%$ electron transmission. A 2.54 $\mathrm{M} \Omega$ resistor creates the collection field between the Frisch grid and the anode with a ratio of electric fields between the drift region and the collection region of 1:2.54, similar to the design used in COSI-FAN-TUTTE [20]. The resistors used in the circuit have a $0.1 \%$ uncertainty to ensure uniform electric fields are generated. The full drift length between the cathode and the Frisch grid is 8.7 $\mathrm{cm}$. The operating drift field is approximately $400 \mathrm{~V} / \mathrm{cm}$. 


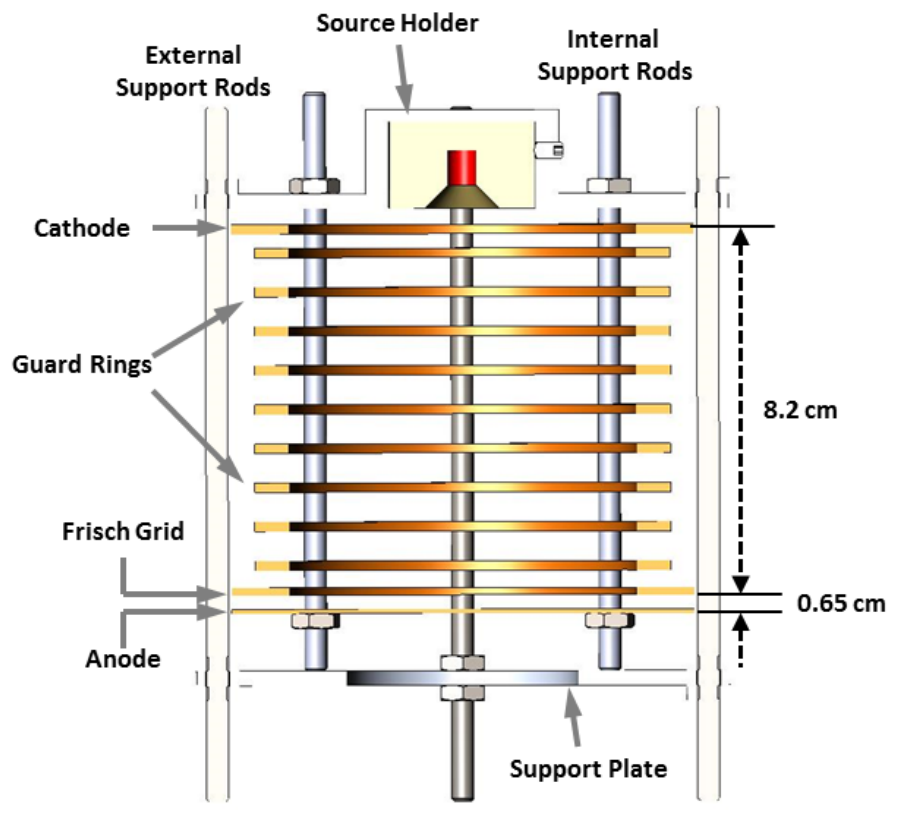

(a)

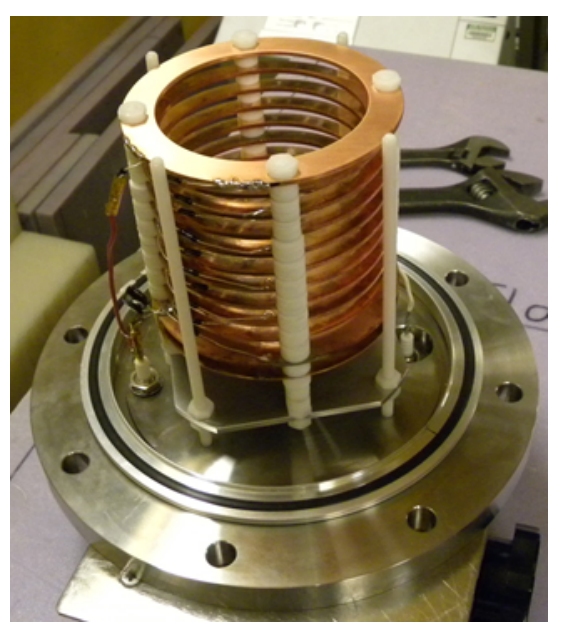

(b)

Figure 2: Parallel ionization chamber for measuring total kinetic energy of fission products. (a) Schematic of ionization chamber components with a check source at the top. The source holder and cathode are replaced by the entrance window during normal operation. (b) Photograph of internal structure of ionization chamber. The fragments enter from the top in this picture. 


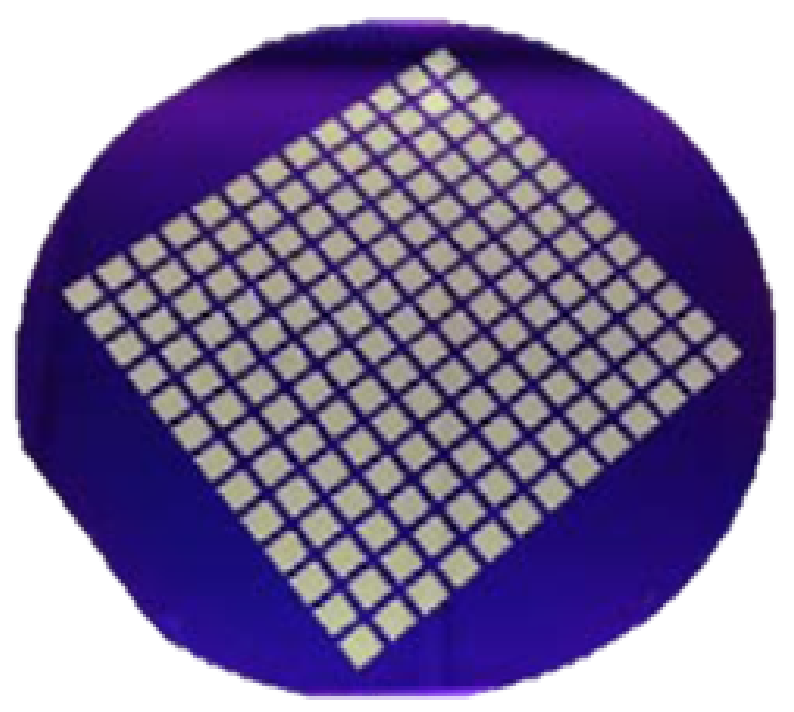

(a)

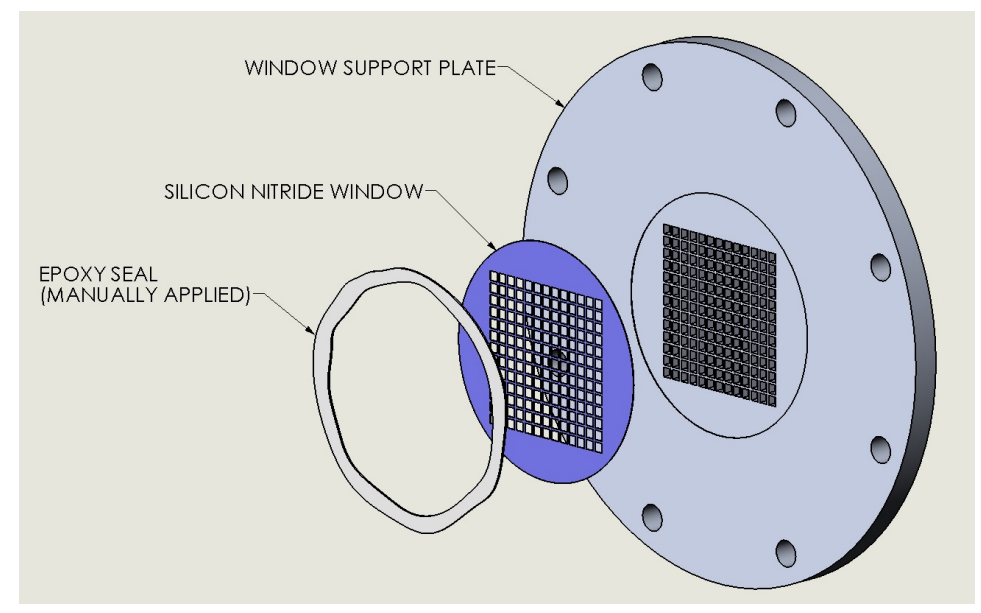

(b)

Figure 3: Silicon nitride entrance window for ionization chamber. (a) Silicon nitride membrane on silicon support wafer. (b) Exploded view of window mounting apparatus and support structure for entrance window. 
The fill gas used is high purity isobutane at 85 Torr $(11.3 \mathrm{kPa})$ for fission products and 200 Torr $(26.7 \mathrm{kPa}$ ) for alpha particles (up to approximately 7 $\mathrm{MeV})$. The gas was continuously exchanged with an approximate flow rate of $14.5 \mathrm{sccm}(0.87 \mathrm{l} / \mathrm{h})$. The continuous flow of gas was necessary to maintain the pulse height of the peaks over the course of data acquisition.

The readout of the ionization chamber is through the anode. The signal is sent directly through an Ortec 142PC preamplifier before entering the acquisition electronics.

\subsection{Acquisition Electronics}

The electronics setup consists of CAEN V1290N time to digital converters (TDCs) with a $40 \mathrm{MHz}$ sampling frequency for the MCP timing signals and the four delay line anode signals for lateral position from each TOF detector and a CAEN VX1724 digitizer with 14-bits and 100 Msamples/s for the energy signals $[21,22]$. The digitizer board uses an on-board trapezoidal filter to extract pulse height in real time [23]. The pulse height of the flat top of the trapezoid and the zero crossing of the second time derivative are saved to disk along with the timing values from the TDCs. The trigger for data collection is based on a logic AND requirement between a "computer ready" signal and a timing signal from the accelerator at LANSCE (labeled as 't0' in far left of Fig. 4) indicating the beginning of the neutron pulse. Once this requirement is met, a 'lookingtime' window is generated with a gate and delay generator and sent to both the digitizer and the TDCs for data acquisition. Figure 4 diagrams the components and logic requirements of the trigger scheme for one arm of SPIDER. The 't0' accelerator timing signal is distributed to all modules in order to reconstruct events on the MCPs and ionization chamber that are coincident in time. At the end of the looking time window the data is read off the electronics with MIDAS-based data acquisition software. [24] 


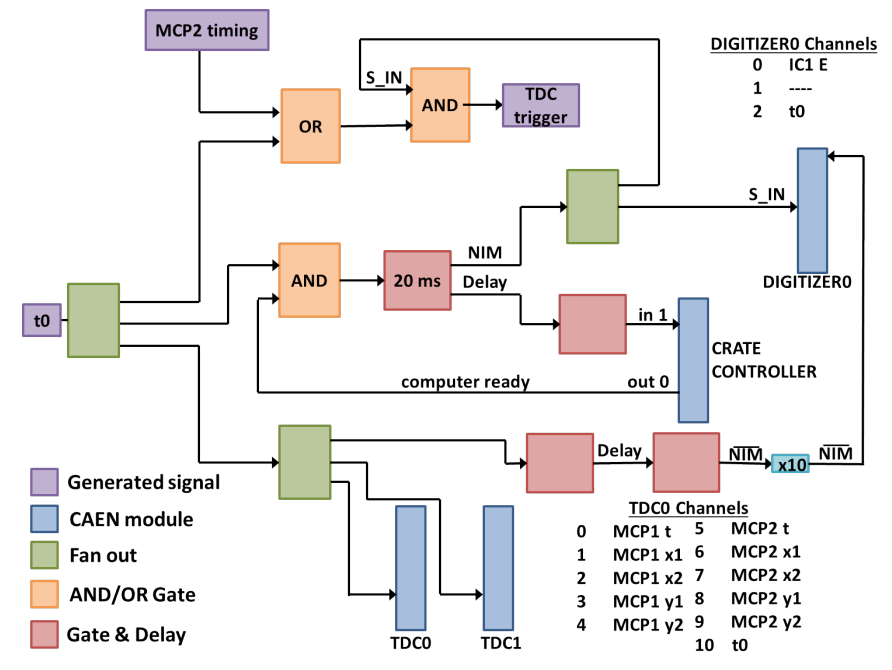

Figure 4: Schematic of data acquisition with trigger setup for one arm of SPIDER.

2.4. 1-arm Set-up

Two timing detectors and one energy detector constitute an arm of SPIDER, shown schematically in Fig. 5. The main vacuum chamber is approximately $32.5 \mathrm{~cm}$ in diameter and $92.5 \mathrm{~cm}$ long, containing the target mounting structure and the timing detectors. The MCPs, electrostatic mirrors, and DLAs are mounted on rails, allowing for a precise selection of the distance between the two timing detectors. This distance defines the nominal flight path length of the fission product as well as the central trajectory. The entrance window of the energy detector is situated directly after the second timing detector, followed immediately by the active detection area of the energy detector mounted to the end of the vacuum chamber.

\section{Characterization and Calibration}

Radioactive sources with well-known characteristics were placed in front of each detector to test, characterize, and calibrate each detector in the SPIDER 


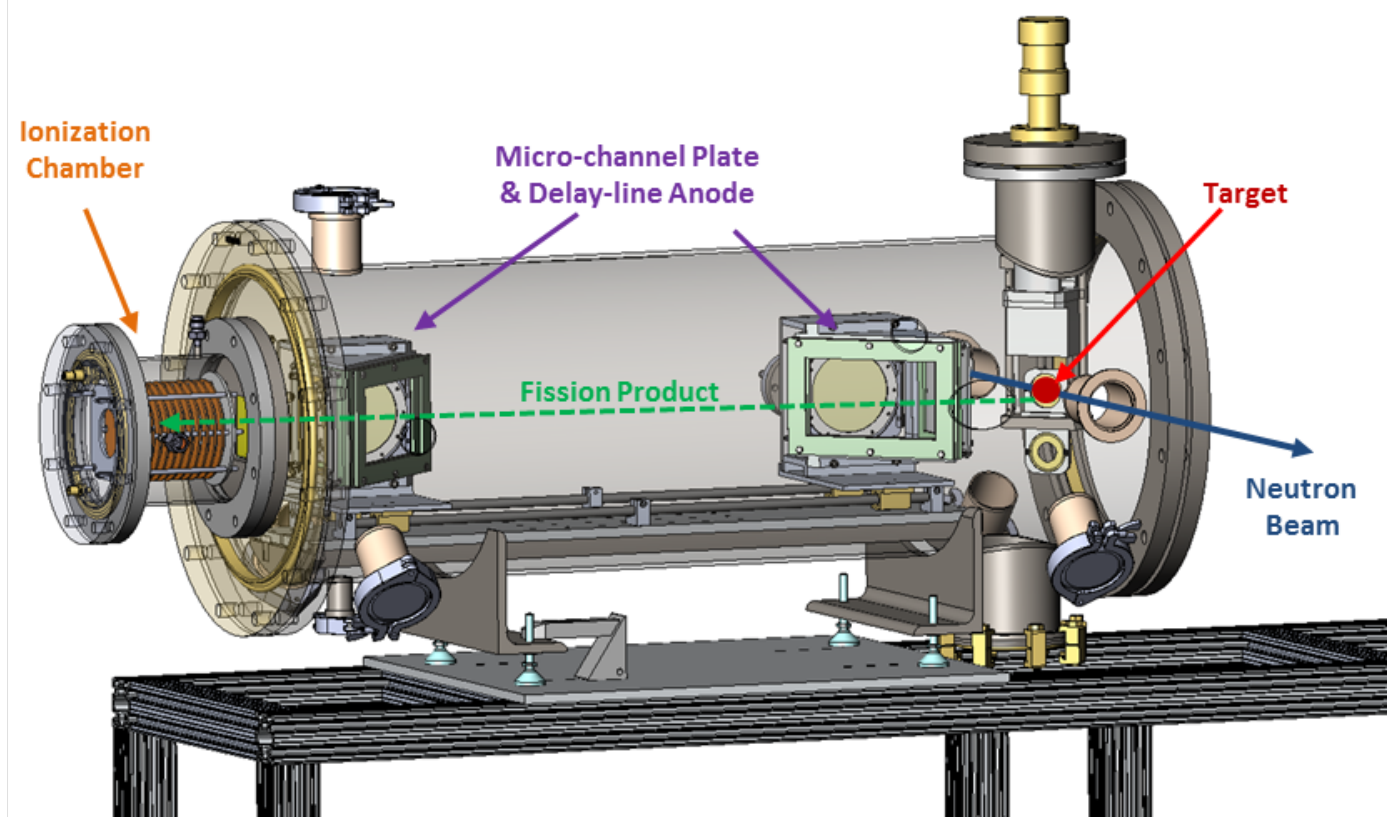

Figure 5: Schematic of one arm setup of SPIDER. Fission product trajectory shown with dashed line moving right to left. 
instrument. Two types of sources were used: a ${ }^{229} \mathrm{Th}$ alpha source and a ${ }^{252} \mathrm{Cf}$ source. The ${ }^{229}$ Th and its decay chain provided 6 alpha lines with well known energies for calibration over a range of energies (for the ionization chamber) and velocities (timing detectors).

\subsection{Time-of-flight and path length detectors}

The high voltage settings for the MCPs and electrostatic mirrors were investigated to determine the optimal values for minimizing channel cross talk, signal noise, and maximize signal-to-noise ratios. The final value of the high voltages $2450 \mathrm{~V}, 2550 \mathrm{~V}$, and $2700 \mathrm{~V}$ for the back, anode holder, and DLAs respectively. These values follow the manufacturer's recommendations for operation of the detectors [25]. After the noise and cross talk were reduced, the ${ }^{229} \mathrm{Th}$ was put in place and the alpha particles' time-of-flight were measured. These measurements were used to determine the timing resolution and linear response of the MCPs. A position mask with $1 \mathrm{~mm}$ holes spaced $1 \mathrm{~cm}$ apart was placed directly in front of the conversion foil in order to determine the position resolution of the DLAs. A resolution of 250 ps was found for the time-of-flight measurement. A lateral position resolution of $2 \mathrm{~mm}$ was also determined. More details about each of these measurements can be found in the Nuclear and Instrumental Methods in Physics Research A paper published for the timing and position detectors of SPIDER [18].

\subsection{Energy detector}

A similar search for optimal operating settings was performed for the ionization chamber. Based on the available drift length $(8.7 \mathrm{~cm}$ between the cathode and Frisch grid) and the chosen fill gas (isobutane), the applied electric field was chosen to stay within the 'plateau' region of the electron drift velocity in order to have stable charge collection even amidst small voltage drifts. The circuit was first tested for discharge points with nonflammable fill gas of P10 


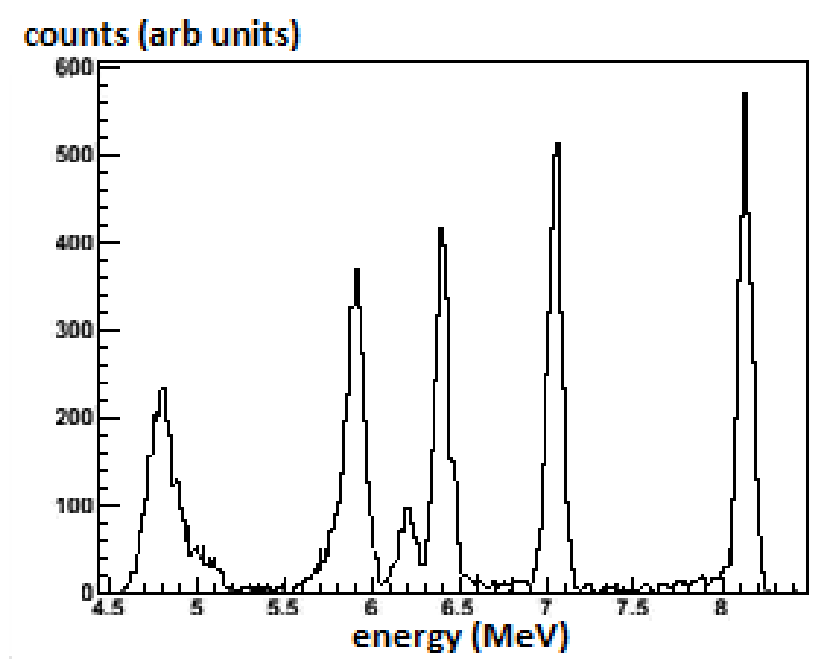

Figure 6: Energy spectrum of alpha particles from ${ }^{229} \mathrm{Th}$ and its decay chain as measured with the ionization chamber of SPIDER. The detector was operated with 200 Torr $(26.7 \mathrm{kPa})$ of isobutane gas flowing continuously and with an applied electric field of approximately $230 \mathrm{~V} / \mathrm{cm}$.

(90\% argon and 10\% methane) prior to operation with isobutane. The ${ }^{229} \mathrm{Th}$ source was also placed inside the IC directly in front of the cathode to determine the energy resolution of the detector. 200 Torr $(26.7 \mathrm{kPa})$ of isobutane gas was required to stop most alpha particles within the active region of the detector. These measurements also allowed for optimization of the trapezoid shaping-parameters used in the on-board pulse height analysis firmware. The major shaping-parameters adjusted were the rise time, flat top width, sampling size, digital gain, and peaking time [26]. Figure 6 shows a spectrum of alpha peaks collected after adjusting the hardware and firmware parameters. As can be seen by the wide peaks, the numerous alpha particles below approximately 7 $\mathrm{MeV}$ were not completely resolved. Therefore these were not used in the calculation of the energy resolution. The FWHM energy resolution for the stopped alpha particles is $1.1 \%$ or $92 \mathrm{keV}$ for $8.376 \mathrm{MeV}$.

The ${ }^{252} \mathrm{Cf}$ source was also placed inside the IC. The operating pressure was 
85 Torr $(11.3 \mathrm{kPa})$ with continual gas flow. Placing the source inside the detector measures the fission spectrum without needing corrections for energy loss through the entrance window. The relative peak heights and peak-to-valley ratios were determined to be similar to previous publications ([27] page 155); an indication that the IC detector was working properly and optimized for fission products.

\section{Signal Processing and Data Analysis}

The first mass yield measurements with SPIDER were taken with one arm of the instrument. An E-v measurement determined the mass of the measured fission products from the spontaneous fission of ${ }^{252} \mathrm{Cf}$. A sample of ${ }^{252} \mathrm{Cf}, 5$ $\mathrm{mm}$ in diameter with a $50 \mu \mathrm{g} / \mathrm{cm}^{2}$ gold cover and an approximate activity of $0.73 \mu \mathrm{Ci}$, was place approximately $3 \mathrm{~cm}$ from the conversion foil of the first or start timing detector. The second or stop timing detector was placed 70.0 $\mathrm{cm}$ downstream, measured from conversion foil to conversion foil. The IC was mounted directly after the stop timing detector with an approximately $6 \mathrm{~cm}$ gap between the conversion foil and the entrance window. Data were collected for all components of the one arm of SPIDER; the two timing MCPs, the two DLAs, and the IC. The following sections describe the data analysis involved and the mass yield results of the measurement.

Data analysis falls into two main categories: calibrations and corrections. The timing and position signals were calibrated using the previously discussed alpha measurements from ${ }^{229} \mathrm{Th}$ and its decay chain. The IC signal was calibrated relative to previous measurements [28, 29] of the average peak energies of the ${ }^{252} \mathrm{Cf}$ energy distribution and corrected for energy loss based on TRIM calculations, a software package for calculating the transport of ions in matter. [30]. 


\subsubsection{Timing and position signals}

The raw and calibrated timing signals for ${ }^{252} \mathrm{Cf}$ are shown in Fig. 7. The timing signals collected were calibrated using the ${ }^{229} \mathrm{Th}$ alpha decay chain data. The known energies and masses of the alpha particles were used to calculate time-of-flight values for the $70.0 \mathrm{~cm}$ flight path length. The range of alpha particles measured enabled the calculation of gain and offset parameters. The raw timing signals shown in Fig. 7(a) varied over time consistent with a shift in raw peak position of the $6.118 \mathrm{MeV}$ energy alpha from ${ }^{252} \mathrm{Cf}$. This shift was accounted for by a time-dependent offset value in the calibration, resulting in figure $7(\mathrm{~b})$.

Eight position signals were collected in addition to the timing spectrum four from each DLA. This placed a small limitation to data analysis as every event did not contain all 8 signals. Future analysis will use the position signals to calculate the path length on an event-by-event basis.

\subsubsection{Energy signals}

The energy signals produced by the ionization chamber are shown in Fig. 9. A representative raw waveform from the IC after pre-amplification, shown in Fig. 8(a), illustrates the clear signal with an excellent signal-to-noise ratio. An example output of the trapezoid-filter waveform from the on-board digitizer firmware is shown in Fig. 8(b). The energy value was determined by the height of the flat top region of the trapezoid shaped waveform. The overshoot at the beginning of the flat top region is a common effect and can be bypassed with firmware settings. The timing values were obtained from the zero-crossing point of the second derivative of the raw waveform. The initial linear calibration parameters for the energy signal were determined by fitting the peaks in the raw spectrum (shown in Fig. 9(a)) with literature values of post neutron-emission peak kinetic energies for the light and heavy fission peaks [28, 29]. Energy loss corrections, calculated with TRIM [30] were necessary to account for energy loss of the fission products in materials prior to measurement in the ionization 


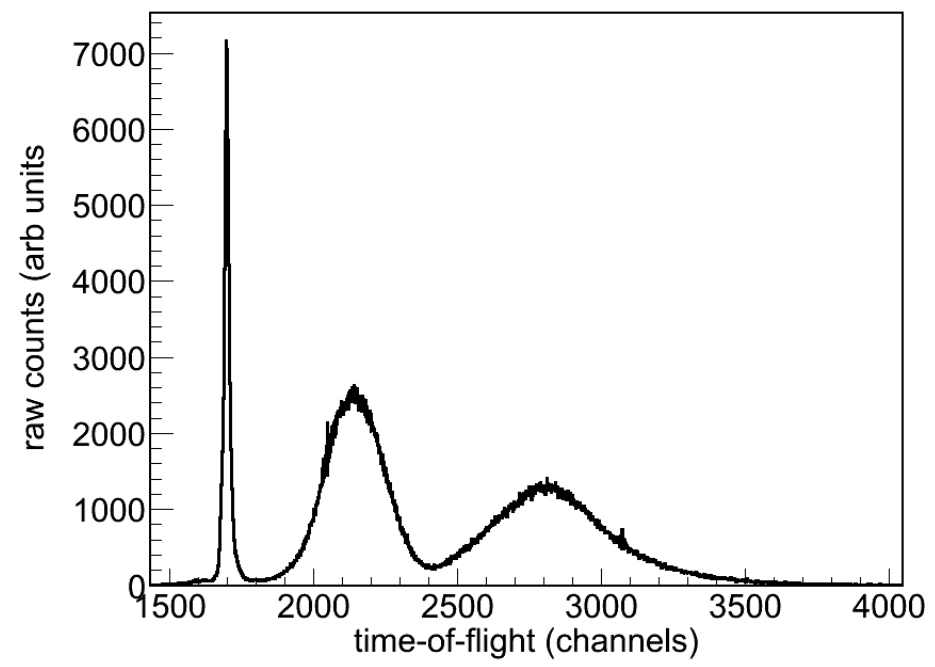

(a)

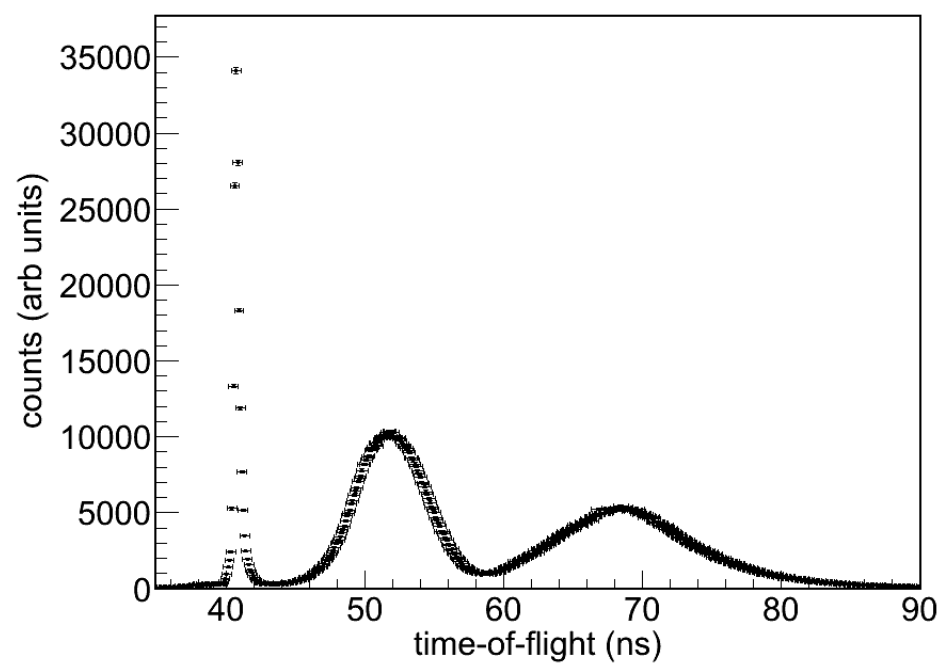

(b)

Figure 7: (a) Raw and (b) calibrated time-of-flight spectra for spontaneous fission fragments from ${ }^{252} \mathrm{Cf}$. Sharp leftmost peak is $6.12 \mathrm{MeV}$ alpha particle emission. 


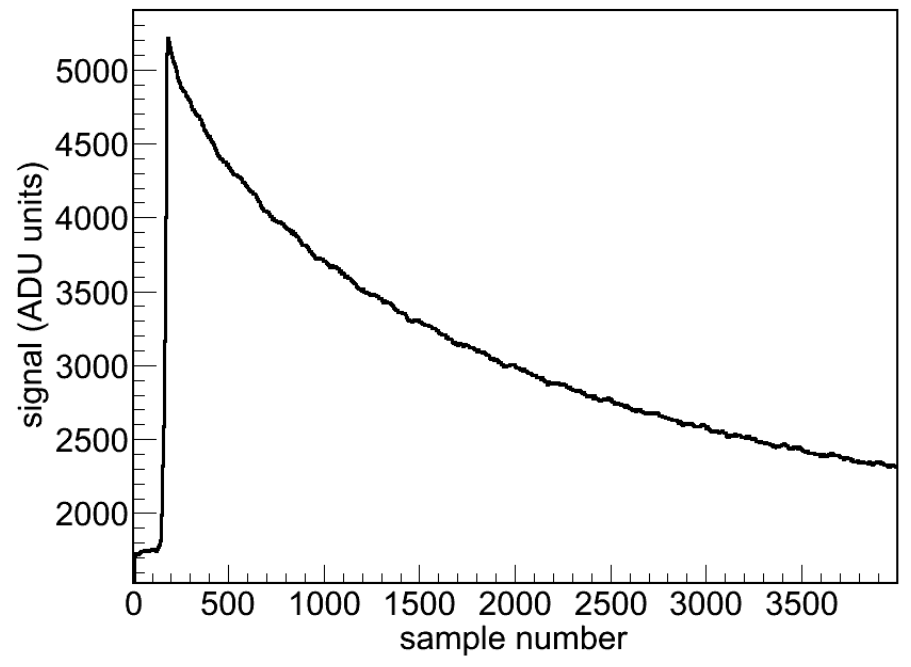

(a)

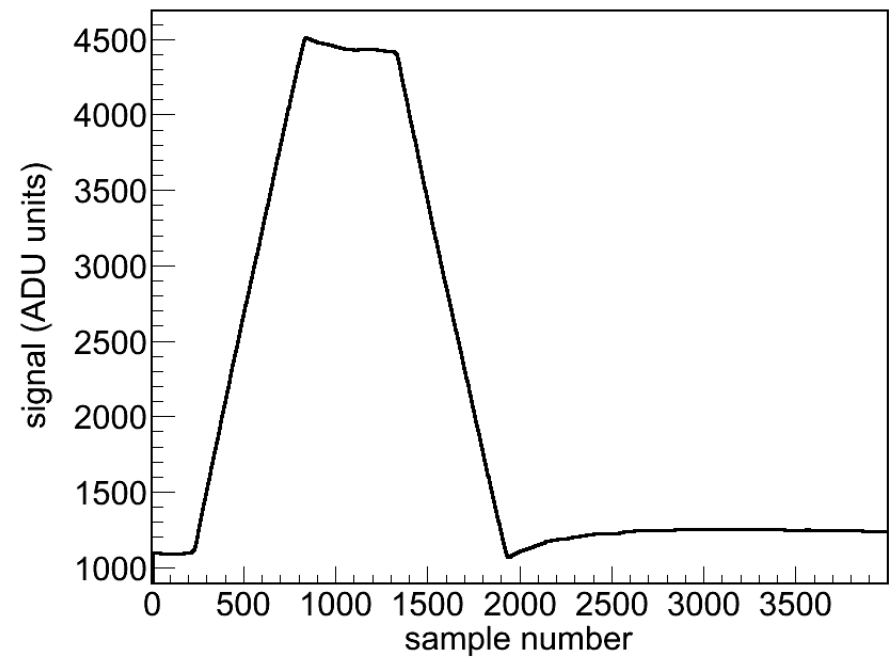

(b)

Figure 8: Example (a) raw waveform and (b) trapezoid outputs of ionization chamber as a function of time. Trapezoid shaped waveform made with on board pulse height analysis firmware. The waveforms were sampled for 4000 channels. 
chamber. The materials upstream of the ionization chamber included a gold foil layer on the ${ }^{252} \mathrm{Cf}$ source, carbon conversion foils in the timing detectors, and the silicon nitride entrance window of the ionization chamber. TRIM calculations estimate the total energy loss through these materials to be $5.5 \mathrm{MeV}$ on average. This energy loss calculation is dependent on initial mass, charge, and energy of the fission products and has a reported uncertainty of 5.8\% [30]. Energy loss was approximated for the entire energy spectrum by a linear fit to calculated energy loss values for a representative set of fission product masses, charges, and initial energies, based on the reported average masses, energies, charges, and neutron emission as a function of mass for the ${ }^{252} \mathrm{Cf}$ fissioning system. [28, 29]. Figure $9(\mathrm{~b})$ is the resulting calibrated energy spectrum.

\section{Results and Discussion}

This section discusses the first results from the SPIDER instrument. The first distributions of energy and velocity for ${ }^{252} \mathrm{Cf}$ fission products are presented. The post-neutron-emission mass distribution of ${ }^{252} \mathrm{Cf}$, determined using the E-v method is also presented with measured mass uncertainties and calculated mass resolution capabilities of SPIDER.

The measured kinetic energy distribution of the fission products is shown in Fig. 9(b). The energy loss corrections for material loss, initially calculated with TRIM, were small - on the order of $5.5 \mathrm{MeV}$. The resulting calibrated and corrected spectrum in Fig. 9(b) is based on these estimates of energy loss. The average kinetic energies of the light and heavy products measured here are 103.5 $\pm 1.8 \mathrm{MeV}$ and $78.7 \pm 1.3 \mathrm{MeV}$.

The velocity distribution of the fission products shown in Fig. 10 is based on the measured time-of-flight of the products and flight path length of $70.0 \mathrm{~cm}$ based on the measured distance between the two timing detectors. The deduced average velocities of the post-neutron-emission ${ }^{252} \mathrm{Cf}$ products were $13.5 \pm 0.1$ $\mathrm{mm} / \mathrm{ns}$ for the light products and $10.13 \pm 0.08 \mathrm{~mm} / \mathrm{ns}$ for the heavy products. 


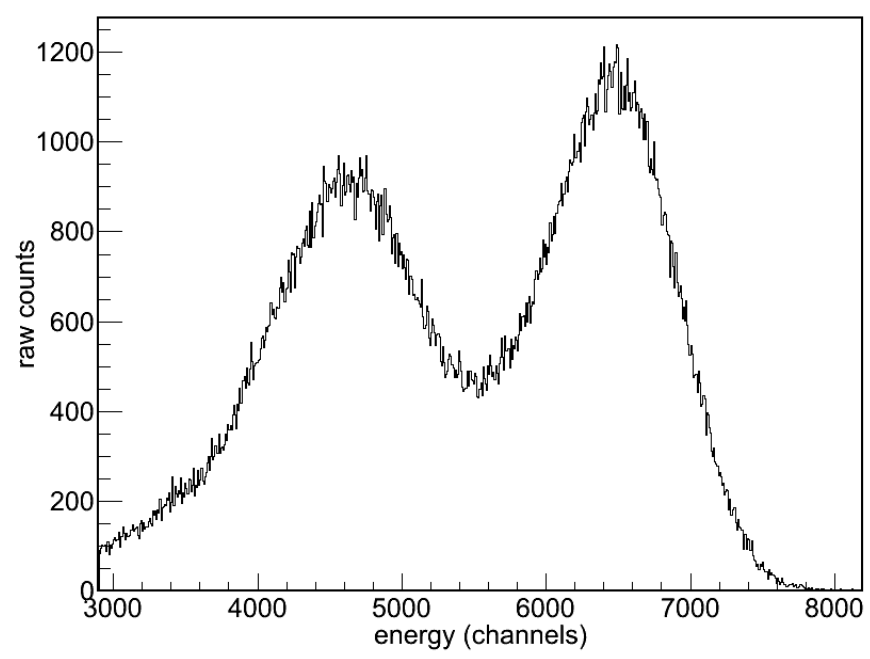

(a)

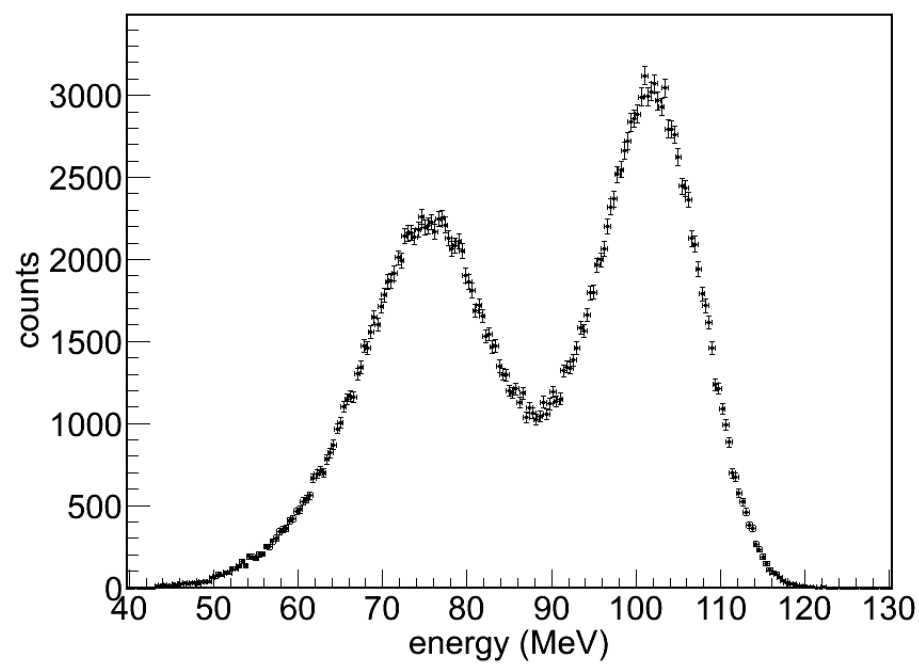

(b)

Figure 9: (a) Raw and (b) calibrated energy spectra for spontaneous fission of ${ }^{252} \mathrm{Cf}$. 


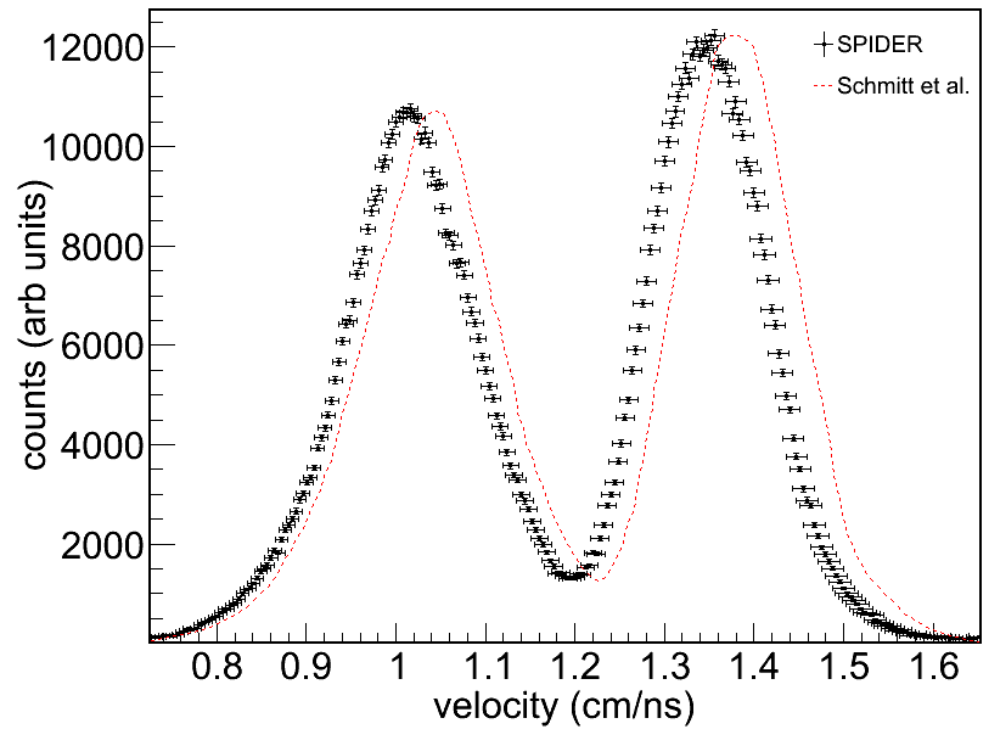

Figure 10: Velocity distribution for post-neutron emission ${ }^{252} \mathrm{Cf}$ fission products measured with SPIDER. Data from Schmitt et al. from Ref. [28]. 


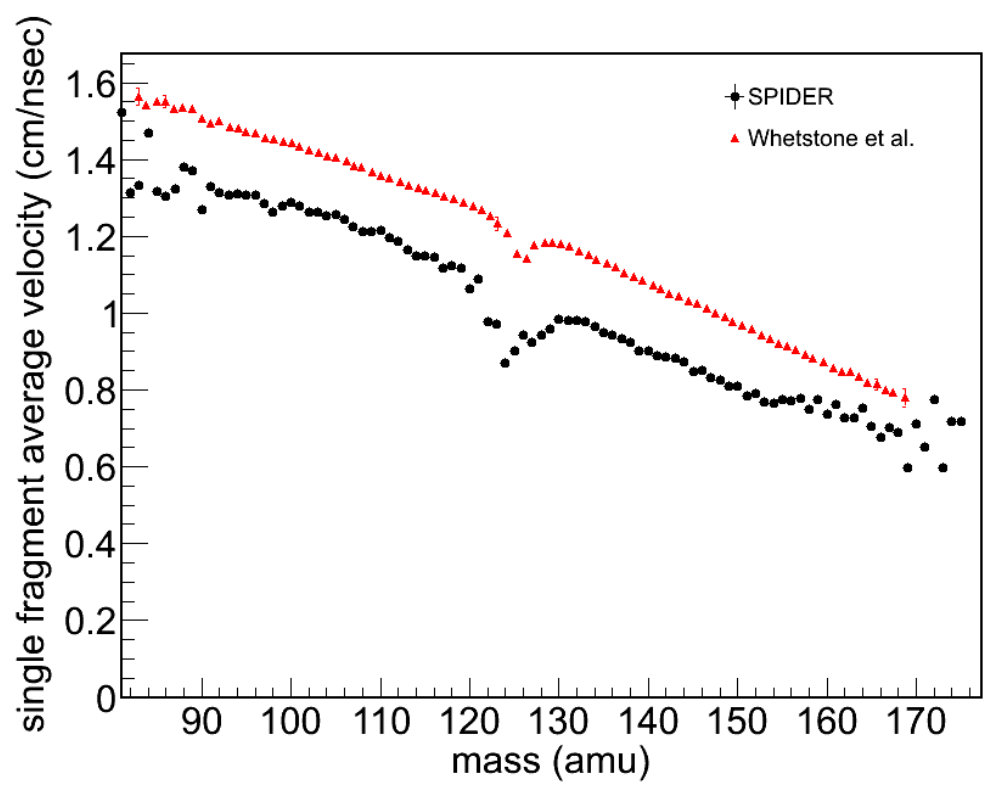

Figure 11: Single fragment average velocity as a function of fragment mass. Data from Whetstone from Ref. [31]. SPIDER data is reported as post-neutron emission data and Whetstone data is reported as pre-neutron emission data.

The average single fragment velocity is plotted in Fig. 11 as a function of mass. The symmetric mass division occurs at the dip in the overall linear regression of the two parameters similar to Fig. 7(a) of [31].

The post-neutron-emission mass yield distribution obtained from this experiment is shown in Fig. 12. The peak values of the light and heavy masses for ${ }^{252} \mathrm{Cf}$ are $107.1 \pm 0.8 \mathrm{amu}$ and $140.7 \pm 1.1 \mathrm{amu}$, respectively. The final energy calibration parameters used to obtain this spectrum were determined by a $\chi^{2}$ minimization search between the measured mass spectrum and the evaluated mass spectrum from England and Rider [32]. The resulting estimate of experimental energy loss for the heavy ion energy peak is approximately $33 \%$ larger than the value obtained from the TRIM calculation. This difference in energy loss is suspected to be a mixture of reported TRIM uncertainties in this mass 
and energy region, uncertainties in material properties, including the molecular composition of the silicon nitride membrane, and the response of the detector for these types of ions, in particular pulse-height defect effects. Further investigation of both the molecular composition of the thin windows and a more thorough calibration of the ionization chamber at a heavy ion facility will constrain these uncertainties in the calibration and correction methods currently being applied to the energy spectrum. The timing calibration described previously, along with energy loss corrections calculated using TRIM, were also used in the calculation of the final mass spectrum shown in Fig. 12. The full integral of the mass result was normalized to $200 \%$.

The mass uncertainties shown for the SPIDER mass distribution were calculated using standard error propagation techniques and included several components. Systematic uncertainties based on calibration and correction parameters used in the analysis of the raw energy and timing signals were the largest contributers to the mass uncertainty. Specifically the linear calibration of the energy and timing measurements contained uncertainties for both the gain and offset terms. The calculation of the velocity uncertainties included the timing calibration uncertainties and the measured uncertainty of the nominal flight path length $(0.1 \%)$. The uncertainty of the energy component also included the uncorrelated uncertainty in the energy loss correction. This uncertainty was set to $5.8 \%$ based on the reported uncertainty in the TRIM calculation for ions of this mass and charge range through materials [30].

The mass result shown in Fig. 12 is compared to the yield results from the mass yield data evaluation by England and Rider in 1993 [32]. This evaluation is the current recommended yield data for spontaneously fissioning ${ }^{252} \mathrm{Cf}$ in ENDF/B-VI. The evaluated yield values range from 66 to 172 amu with yield uncertainties approximated by reported experimental variances. The two yield distributions are in good agreement with each other, particularly at the peak of the light and heavy mass yields. It should be noted that no broadening of the evaluation data was necessary to make a comparison to the SPIDER result. As more statistics are collected with SPIDER on ${ }^{252} \mathrm{Cf}$ and other fissioning systems, 


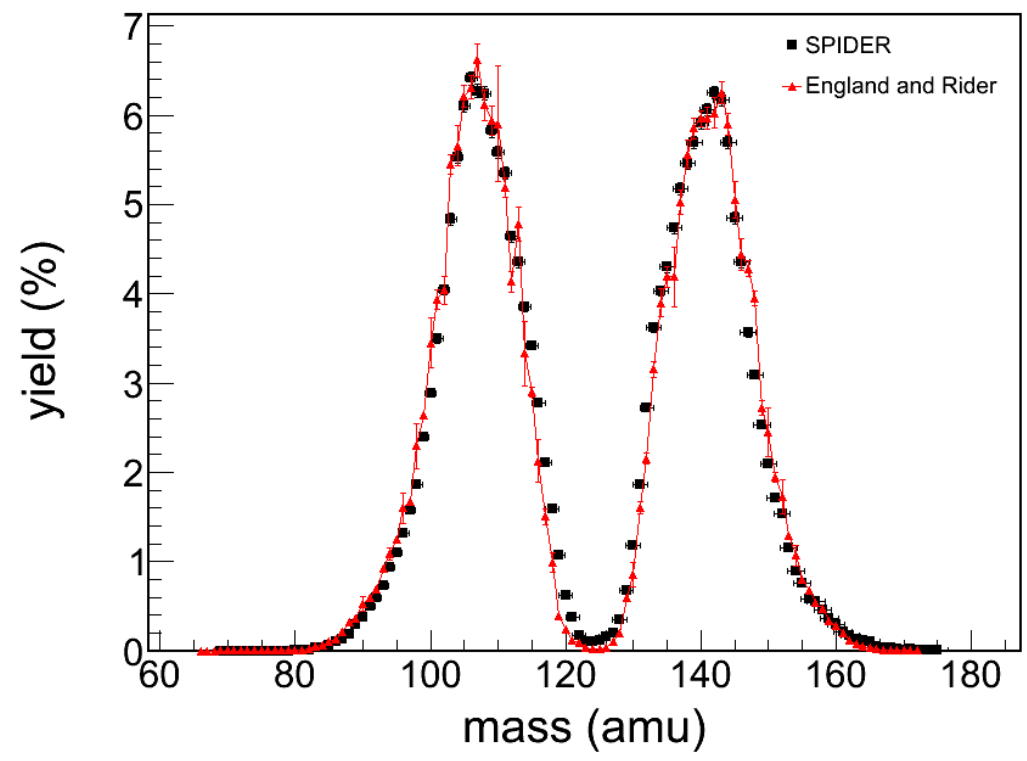

Figure 12: Mass yield distribution of spontaneously fissioning ${ }^{252}$ Cf. SPIDER data shown as filled black squares, England and Rider evaluation [32] shown as filled red triangles and solid red line.

mass regions with rapidly changing yields will be thoroughly investigated.

The current mass resolution capabilities of SPIDER have been calculated based on the individual measurement resolutions for energy, time-of-flight, and path length and an energy resolution component from calculated energy straggling through material. The measured energy resolution of $1.1 \%$ for alpha particles is similar to reported alpha resolutions for optimization ionization chambers [33]. This resolution was reduced by a factor of two to $0.55 \%$ for fission fragments based on the assumption of optimized the SPIDER ionization chamber and reported energy resolutions of fission fragments in similarly designed and optimized detectors [20]. A second energy resolution component was added into the resolution equation discussed earlier to approximate the limit in resolution based on energy straggling of the fragments through the materials. This 
resolution is dependent on fragment species and was estimated using TRIM calculations of energy straggling for mass 107 and mass 141 to be $0.25 \%$ and $0.47 \%$, respectively. The measured resolution for time-of-flight was $0.5 \%$. Finally, the path length resolution of $0.03 \%$ was based on the extrapolated position resolution over the nominal $700.0 \mathrm{~mm}$ path length. The four components of the resolution were added in quadrature to determine the mass resolution of the mass peak values: $1.2 \mathrm{amu}$ at $107 \mathrm{amu}$ and $1.7 \mathrm{amu}$ at $141 \mathrm{amu}$.

\section{Future Work}

The goal of measuring coincidence fission products with the $2 \mathrm{E}-2 \mathrm{v}$ method has progressed with the construction and initial testing of a two-arm system for SPIDER. The coincidence measurements will allow for determination of several fission properties including total kinetic energy (TKE) of the products, TKE vs mass studies, and neutron emission as a function of mass. The fully constructed and assembled SPIDER two-arm system shown in Fig. 13 is installed currently at a Lujan Center flight path at the LANSCE facility. Source tests of the system are ongoing as are additions to software to accommodate the additional data. The Lujan Center provides a thermal neutron spectrum and will be used to measure neutron-induced fission mass yields and coincidence studies, starting with ${ }^{235} \mathrm{U}$ and ${ }^{239} \mathrm{Pu}$ isotopes. Fast neutron-induced yields will be studied at the WNR facility. The size of the detectors was chosen to accommodate the small shift in the reaction kinematics with increasing excitation energy and is not expected to decrease the efficiency of SPIDER.

\section{Summary}

The energy-velocity method of measuring mass yields of fission products has been successfully applied using the newly constructed SPIDER instrument. The individual detector components for measuring time-of-flight, path length, and energy have met resolution requirements necessary for meeting the goal of one atomic mass unit resolution. The first results reported for mass yields of $\mathrm{Cf}$ 


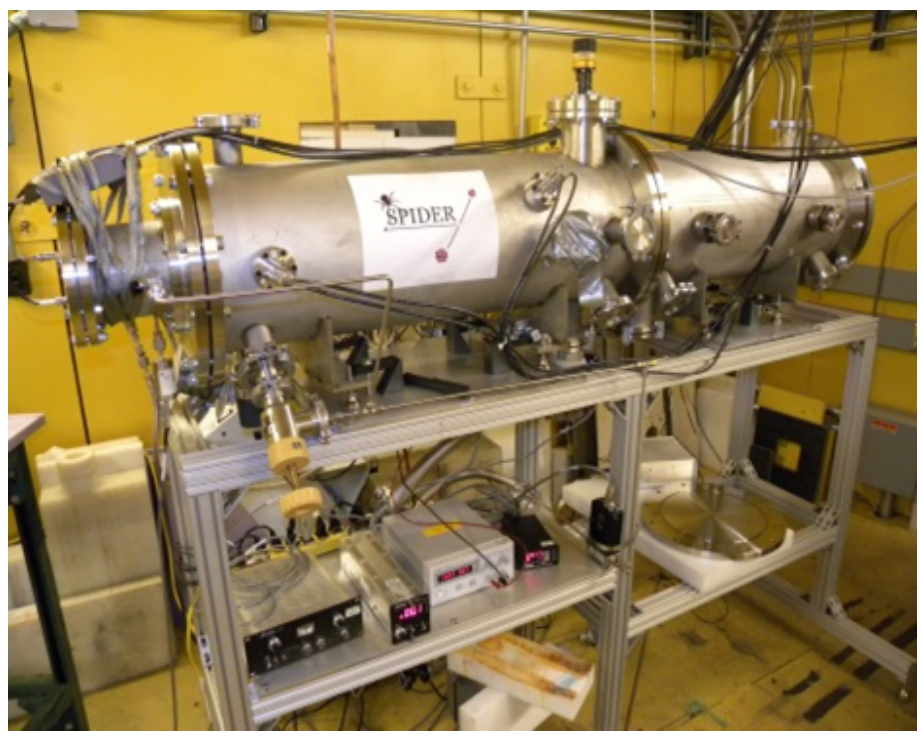

Figure 13: Picture of two-arm setup of SPIDER installed on flight path 12 at the Lujan Center at the LANSCE facility.

fission products are consistent with an average mass yield resolution of $1 \mathrm{amu}$. Future work will consist of coincidence product measurements with the $2 \mathrm{E}-2 \mathrm{v}$ method with a focus on neutron-induced fission of actinide species.

\section{Acknowledgements}

This work benefited from the use of the LANSCE accelerator facility and was performed under the auspices of the US Department of Energy by Los Alamos Security, LLC under contract DE-AC52-06NA25396.

\section{References}

[1] O. Hahn, F. Strassmann, Concerning the existence of alkaline earth metals resulting from neutron irradiation of uranium, Die Naturwissenschaften 27 (1939a) 11-15. 
[2] O. Hahn, F. Strassmann, Proof of the formation of active isotopes of barium from uranium and thorium irradiated with neutrons; proof of the existence of more active fragments produced by uranium fission, Die Naturwissenschaften 27 (1939b) 89-95.

[3] L. Meitner, O. Frisch, Disintegration of uranium by neutrons: a new type of nuclear reaction, Nature 143 (1939) 239-240.

[4] H. Anderson, E. Booth, J. Dunning, E. Fermi, G. Glasoe, F. Slack, The Fission of Uranium, Physical Review 55 (1939) 511.

[5] C. Budtz-Jorgensen, H.-H. Knitter, C. Straede, F.-J. Hambsch, R. Vogt, A twin ionization chamber for fission fragment detection, Nuclear Instruments and Methods Section A 258 (2) (1987) 209-220.

[6] H. Faust, P. Geltenbort, F. Gönnenwein, A. Oed, Determination of the isobaric elemental yields in velocity selected fission products, Nuclear Instruments and Methods 193 (1982) 577-580.

[7] P. Geltenbort, F. Gönnenwein, A. Oed, Precision measurements of mean kinetic energy release in thermal-neutron-induced fission of ${ }^{233} \mathrm{U},{ }^{235} \mathrm{U}$, and ${ }^{239} \mathrm{Pu}$, Radiation Effects 93 (1-4) (1986) 57-60.

[8] P. Geltenbort, F. Gönnenwein, A. Oed, P. Perrin, Mass spectrometry of fission fragments by simultaneous energy and time-of-flight measurements, Radiation Effects 95 (1-4) (1986) 325-330.

[9] N. Boucheneb, P. Geltenbort, M. Aschar, G. Barreau, T. Doan, F. Gönnenwein, B. Leroux, A. Oed, A. Sicre, High resolution measurements of mass, energy and nuclear charge correlations for ${ }^{229} \mathrm{Th}\left(\mathrm{n}_{t h}, \mathrm{f}\right)$ with the cos fan tutti spectrometer, Nuclear Physics A 502 (1989) 77-93.

[10] A. Oed, P. Geltnebort, R. Brissot, F. Gönnenwein, P. Perrin, E. Aker, D. Engelhardt, A mass spectrometer for fission fragments based on timeof-flight and energy measurements, Nuclear Instruments and Methods in Physics Research 219 (1984) 569-574. 
[11] A. Sicre, G. Barreau, A. Boukellal, F. Caïtucoli, T. Doan, B. Leroux, High resolution study of $\mathrm{U} 235\left(\mathrm{n}_{t h}, \mathrm{f}\right)$ and $\mathrm{Th} 229\left(\mathrm{n}_{t h}, \mathrm{f}\right)$ with cosi fan tutte mass spectrometer, Radiation Effects 93 (1-4) (1986) 65-68.

[12] N. Boucheneb, M. Asghar, G. Barreau, T. Doan, B. Leroux, A. Sicre, P. Geltenbort, A. Oed, A high-resolution multi-parametric study of ${ }^{239} \mathrm{Pu}\left(\mathrm{n}_{t h}, \mathrm{f}\right)$ with the Cosi-Fan-Tutte spectrometer, Nuclear Physics A 535 (1) (1991) 77-93.

[13] P. Schillebeeckx, C. Wagemans, P. Geltenbort, F. Gönnenwein, A. Oed, Investigation of mass, charge and enegry of ${ }^{241} \mathrm{Pu}\left(\mathrm{n}_{t h}, \mathrm{f}\right)$ fragments with the Cosi-Fan-Tutte spectrometer, Nuclear Physics A 580 (1994) 15-32.

[14] M. Chadwick, Future Challenges for Nuclear Data Research in Fission, Journal of Korean Physical Society 59 (2) (2011) 752-754.

[15] H. Britt, J. Dairiki, R. Lougheed, D. McNabb, S. Prussin, Review of the Status of Cumulative Fission Yields from $239 \mathrm{Pu}(\mathrm{n}, \mathrm{f})$ of Interest to $\mathrm{Nu}-$ clear Forensics, Technical Report, Lawrence Livermore National Laboratory, LLNL-TR-458777.

[16] J. Lestone, Energy Dependence of Plutonium Fission-Product Yields, Nuclear Data Sheets 112 (12) (2011) 3120-3134.

[17] P. W. Lisowski, K. F. Schoenberg, The Los Alamos Neutron Science Center, Nuclear Instruments and Methods in Physics Research A 562 (2) (2006) 910-914.

[18] C. Arnold, F. Tovesson, K. Meierbachtol, T. Bredeweg, M. Jandel, H. Jorgenson, A. Laptev, G. Rusev, D. Shields, M. White, R. Blakeley, D. Mader, A. Hecht, Development of position-sensitive time-of-flight spectrometer for fission fragment research, Nuclear Instruments and Methods in Fission Research A 764 (2014) 53-58.

[19] High-Q $\mathrm{Si}_{3} \mathrm{~N}_{4}$ Membrane Windows, http://www.norcada.com/. 
[20] A. Oed, P. Geltenbort, F. Gönnenwein, T. Manning, D. Souque, High resolution axial ionization chamber for fission products, Nuclear Instruments and Methods in Physics Research A 205 (3) (1983) 451-453.

[21] V1290A/N Technical Information Manual, www.caen.it, revision Date: 15 July 2012.

[22] V1724 Technical Information Manual, www. caen. it, revision Date: 30 June 2014.

[23] V. Jordanov, G. Knoll, Digital synthesis of pulse shapes in real time for high resolution radiation spectroscopy, Nuclear Instruments and Methods in Physics Research A 345 (2) (1994) 337-345.

[24] MIDAS Wiki, midas.triumf.ca, accessed numerous times 2012-2014.

[25] MCP Delay Line Detector Manual, www.roentdek.com, Version 11.0.1403.1.

[26] DPHA User Manual, www.caen.it, revision Date: 3 June 2013.

[27] G. Knoll, Radiation Detection and Measurement, Third Edition, John Wiley \& Sons, Hoboken, New Jersey, 1999.

[28] H. Schmitt, W. Kiker, C. Williams, Precision Measurement of Correlated Energies and Velocities of ${ }^{252} \mathrm{Cf}$ Fission Fragments, Physical Review 137 (4B) (1965) 837-847.

[29] H. Henschel, A. Kohnle, H. Hipp, F. Gönnenwein, Absolute measurement of velocities, masses and energies of fission fragments from californium-252 (sf), Nuclear Instruments and Methods in Physics Research 190 (1981) $125-134$.

[30] J. Ziegler, M. Ziegler, J. Biersack, SRIM - The stopping and range of ions in matter (2010), Nuclear Instruments and Methods in Physics Research B 268 (11-12) (2010) 1818-1823. 
[31] S. L. Whetstone Jr., Coincident Time-of-Flight Measurements of the Velocities of $\mathrm{Cf}^{252}$ Fission Fragments, Physical Review 131 (3) (1963) 1232-1243.

[32] T. England, B. Rider., Evaluation and Compilation of Fission Product Yields 1993, Technical Report, Los Alamos National Laboratory, LA-UR94-3106, ENDF-349.

[33] H. Fulbright, Ionization chambers, Nuclear Instruments and Methods 162 (1979) 21-28. 